\title{
PARÂMETROS EFICIENTES DO POSICIONAMENTO ADEQUADO DA PONTA DA AGULHA DE VERESS DURANTE O ESTABELECIMENTO DE PNEUMOPERITÔNIO
}

\author{
RELIABLE GUIDELINES FOR ADEQUATE POSITIONING OF VERESS NEEDLE ON THE \\ STABLISHMENT OF THE PNEUMOPERITONEUM
}

\author{
Otávio Cansanção de Azevedo'; João Luiz Moreira Coutinho Azevedo, ECBC-SP²; \\ Albino Augusto Sorbello ${ }^{3}$; Gustavo Peixoto Soares Miguel ${ }^{4}$; \\ Rodrigo Santa Cruz Guindalini5; Antônio Cláudio de Godoy ${ }^{6}$
}

\begin{abstract}
RESUMO: Objetivo: Avaliar a possibilidade do estabelecimento de parâmetros fidedignos do adequado posicionamento da ponta da agulha de Veress no interior da cavidade peritoneal durante o estabelecimento do pneumoperitônio. Método: Em 100 pacientes selecionados a ponta da agulha de Veress foi introduzida na cavidade peritoneal e o insuflador foi programado para fluxo de $1,2 \mathrm{~L} / \mathrm{min}$ e pressão máxima final para $12 \mathrm{mmHg}$. No início da insuflação e a cada 20 segundos a pressão intraperitoneal (PI) e o total do volume injetado até aquele momento (TVI) eram registrados. Os dados foram tratados por correlações estatísticas entre momentos e PI, e momentos e TVI. Também foi estabelecida a previsão dos valores de PI e TVI no final de cada um dos quatro primeiros minutos de insulflação, utilizando-se os modelo estimados: $\mathbf{P I}=2,3083+0,0266 \times$ tempo $+8,3 \times 10^{-5} \times$ tempo $^{2}-2,44 \times 10^{-7} \times$ tempo $;$ TVI $=0,813$ + 0,0157 x tempo. Resultados: A PI e o TVI mostraram correlação entre momentos pré-estabelecidos da criação do pneumoperitônio, sendo constatado um ajuste forte: $\mathbf{P I}=-2 \mathrm{E}-07 \times \mathrm{xtempo}^{3}+8 \mathrm{E}-05 \mathrm{x}$ tempo $\mathrm{o}^{2}+0,0266 \mathrm{x}$ tempo $+2,3083$, com coeficiente de explicação $\left(\mathrm{R}^{2}\right)=0,8011$; TVI $=0,0157$ x tempo $+0,1813$, com $\mathrm{R}^{2}=0,9604$. A previsão de PI e TVI mostrou: $\mathbf{P I}(\mathrm{mmHg}): 1 \mathrm{~min}=4,15$; $2 \mathrm{~min}=6,27 ; 3 \mathrm{~min}=8,36 ; 4 \mathrm{~min}=10,10$ e TVI(L): $1 \mathrm{~min}=1,12 ; 2 \mathrm{~min}=2,07 ; 3 \mathrm{~min}=3,01 ; 4 \mathrm{~min}=3,95$. Conclusão: Parâmetros fidedignos para PI e TVI , quando a ponta da agullha de Veress se encontra na cavidade peritoneal, em dados momentos da insuflação podem ser estabelecidos durante a criação do penumoperitônio (Rev. Col. Bras. Cir. 2006; 33(4): 220-223).
\end{abstract}

Descritores: Laparoscopia/ efeitos adversos; Procedimentos cirúrgicos operatórios; Pneumoperitônio artificial; Punções/ métodos; Punções/ efeitos adversos.

\section{INTRODUÇÃO}

As lesões dos grandes vasos constituem a causa principal de morte intra-operatória em procedimentos laparoscópicos ${ }^{1}$. Há na literatura numerosos casos de lesão de grandes vasos com agulha de Veress, que mostram toda a dramaticidade da situação e a dificuldade de se fazer o diagnóstico correto da complicação, devido, principalmente, à posição retroperitoneal dos vasos ${ }^{2-10}$.

Para informação do cirurgião durante a insuflação, é interessante considerar que os níveis da pressão intraperitoneal (PI) e o montante do volume injetado (VI) em cada momento têm caráter objetivo, e seus valores podem ser correlacionados com a situação real da ponta da agulha de Veress na topografia abdominal em determinado instante.

Estudos visando à padronização de paradigmas objetivos e confiáveis que possam indicar o adequado posicionamento da ponta da agulha de Veress no interior da cavidade peritoneal em determinado momento da criação do pneumoperitônio seriam de grande valia para a orientação do cirurgião laparoscopista. Tais pesquisas existem em suínos ${ }^{11}$, mas não em humanos.

Esta pesquisa objetiva estabelecer valores fidedignos com teor preditivo para os níveis de pressão intraperitoneal

1. Médico-Assistente do Serviço de Gastroenterologia Cirúrgica do Hospital do Servidor Público do Estado de São Paulo; Doutorando do Programa de Pós-Graduação em Cirurgia e Experimentação da Universidade Federal de São Paulo (UNIFESP); Pesquisador do Grupo de Videocirurgia do $\mathrm{CNPq} / \mathrm{UNIFESP}$.

2. Professor Adjunto Mestre e Doutor do Departamento de Cirurgia e Responsável pelo Setor de Videocirurgia da Disciplina de Técnica Operatória e Cirurgia Experimental (TOCE) da UNIFESP; Professor Orientador do Programa de Pós-Graduação em Cirurgia e Experimentação da UNIFESP; Ex-Professor Visitante da Universidade de Lyon, França; Líder do Grupo de Pesquisa em Videocirurgia do CNPq/UNIFESP.

3. Médico-Assistente do Serviço de Gastroenterologia Cirúrgica e Chefe do Setor de Videocirurgia do Hospital do Servidor Público do Estado de São Paulo; Professor Colaborador do Setor de Videocirurgia da TOCE-UNIFESP e do Programa de Pós-Graduação em Cirurgia e Experimentação da UNIFESP; Pesquisador do Grupo de Videocirurgia do CNPq/UNIFESP.

4. Doutorando do Programa de Pós-Graduação em Cirurgia e Experimentação da Universidade Federal de São Paulo (UNIFESP); Pesquisador do Grupo de Videocirurgia do CNPq/UNIFESP.

5. Aluno de Graduação da Escola Paulista de Medicina - UNIFESP.

6. Diretor do Serviço de Gastroenterologia Cirúrgica do Hospital do Servidor Público do Estado de São Paulo.

Recebido em 20-03-06

Aceito para publicação em 24-04-06

Conflito de interesses: nenhum

Fonte de financiamento: nenhuma

Trabalho realizado no Serviço de Gastroenterologia Cirúrgica do Hospital do Servidor Público do Estado de São Paulo (HSPE) e no Setor de Videocirurgia da Disciplina de Técnica Operatória e Cirurgia Experimental (TOCE), Departamento de Cirurgia da Universidade Federal de São Paulo (UNIFESP), Programa de Pós-Graduação em Cirurgia e Experimentação. 
(PI) e os volumes injetados (VI) em determinados momentos do decorrer do processo de estabelecimento do pneumoperitônio mediante punção com agulha de Veress, em pacientes selecionados.

\section{MÉTODO}

Esta pesquisa foi aprovada pelo Comitê de Ética em Pesquisa do Instituto de Assistência Médica ao Servidor Público Estadual (protocolo n ${ }^{\circ}$ 045/03) e pelo Comitê de Ética em Pesquisa da Universidade Federal de São Paulo (protocolo ${ }^{\circ}$ 1405/03). Os dados demográficos da amostra estão explicitados na Tabela 1 .

Foram incluídos 100 pacientes consecutivamente agendados para serem submetidos a procedimentos videolaparoscópicos no Serviço de Gastroenterologia Cirúrgica do Hospital do Servidor Público do Estado de São Paulo (HSPE), maiores que 18 anos, não-obesos (Índice de Massa Corpórea - IMC - menor que $30 \mathrm{~kg} / \mathrm{m}^{2}$ ), com saco peritoneal não cirurgicamente violado nem histórico de peritonite pregressa.

Foi ministrado $0,1 \mathrm{mg} / \mathrm{Kg}$ de midazolam trinta minutos antes do ato anestésico. A indução foi realizada com $2 \mathrm{mg} /$ $\mathrm{Kg}$ de propofol e 0,5 mcg/Kg de fentanil, e a curarização com $0,5 \mathrm{mg} / \mathrm{kg}$ de atracúrio. Os pacientes foram submetidos a anestesia geral com ventilação controlada mecânica. Foi introduzida uma sonda até o estômago e aspirado o seu conteúdo. O insuflador foi programado para fluxo de $1,2 \mathrm{~L} / \mathrm{min}$ e pressão máxima final de $12 \mathrm{mmHg}$. No início da insuflação e a

Tabela 1 - Estatística descritiva dos dados demográficos dos pacientes da amostra.

\begin{tabular}{|c|c|}
\hline . Parâmetros & $(n=100)$ \\
\hline \multicolumn{2}{|l|}{ Idade (anos) } \\
\hline média (dp) & $53,7 \quad(13,1)$ \\
\hline mínimo-máximo & $27-77$ \\
\hline \multicolumn{2}{|l|}{ Sexo $-n(\%)$} \\
\hline Feminino & 58 \\
\hline Masculino & 42 \\
\hline \multicolumn{2}{|l|}{$\mathrm{IMC}\left(\mathrm{kg} / \mathrm{m}^{2}\right)$} \\
\hline média (dp) & $25,4 \quad(2,4)$ \\
\hline mínimo-máximo & $20,6-29,7$ \\
\hline \multicolumn{2}{|l|}{ Altura (m) } \\
\hline média (dp) & $1,64 \quad(0,09)$ \\
\hline mínimo-máximo & $1,45-1,87$ \\
\hline \multicolumn{2}{|l|}{ Peso $(\mathrm{kg})$} \\
\hline média (dp) & $(9,8)$ \\
\hline mínimo-máximo & $49,5-90,0$ \\
\hline \multicolumn{2}{|l|}{ Intervenção - n (\%) } \\
\hline Colecistectomia & 80 \\
\hline Fundocardioplastia & 9 \\
\hline Herniorrafia ing bilateral & 3 \\
\hline Herniorrafia ing unilateral & 8 \\
\hline
\end{tabular}

$n=$ número de pacientes $; d p=$ desvio padrão; $\%=$ porcentagem; ing = inguinal. cada 20 segundos a pressão intraperitoneal (PI) e o total do volume injetado até aquele momento (TVI) eram registrados. Os dados foram tratados por correlações estatísticas entre os momentos e PI, e os momentos e TVI. Também foi estabelecida a previsão dos valores de PI e TVI no final de cada um dos quatro primeiros minutos de insulflação, utilizando-se os modelo estimados: $\mathbf{P I}=2,3083+0,0266 \times$ tempo $+8,3 \times 10^{-5} \times$ tempo $^{2}-2,44 \times 10^{-7} \times$ tempo $^{3} ; \mathbf{T V I}=0,813+0,0157 \times$ tempo. Após a punção com agulha de Veress foram utilizadas provas preconizadas para determinar se a ponta da agulha estava no interior do saco peritoneal ${ }^{11}$ e no início da insuflação foi feita a prova da pressão inicial ${ }^{11}$. Com todas essas provas positivas, prosseguia-se com a insuflação de gás carbônico, registrando-se as variações da PI e do montante do VI a partir do momento zero e a cada vinte segundos, até que a PI máxima programada $(12 \mathrm{mmHg})$ fosse atingida, ocasião em que o tempo de duração da insuflação era registrado.

Os dados obtidos foram submetidos a testes estatísticos. As variáveis qualitativas foram representadas por freqüência absoluta (n) e relativa (\%) e as quantitativas por média, desvio padrão (d.p.), e valores mínimo e máximo. Foram utilizados os modelos de regressão polinomiais estimados para volume e pressão em função do tempo.

Para verificar se o modelo de regressão ajustado é adequado ao conjunto de dados, foram estudadas as discrepâncias entre os valores observados em cada tempo e aqueles ajustados pelo modelo. Essas discrepâncias, denominadas resíduos, são as quantidades que a equação de regressão não conseguiu explicar, ou pelo efeito de variáveis externas omitidas no modelo ou pela própria variabilidade natural entre os indivíduos. Por essas análises investigamos a eventual existência de "outliers" que pudessem provocar alterações nas curvas estimadas, bem como ajuste adequado do modelo no que se refere às suposições feitas no modelo adotado.

No caso das correlações serem positivas, serão utilizadas as equações de previsão da PI e do VI, que serão deduzidas das curvas de regressão nos tempos de 1, 2, 3 e 4 minutos de duração da insuflação.

\section{RESULTADOS}

A pressão mostrou correlação positiva entre momentos pré-estabelecidos da criação do pneumoperitônio (Gráfico 1) e a curva mostra um ajuste forte, com coeficiente de explicação igual a 0,80 (pressão $=-2 \mathrm{E}-07 \times$ tempo $^{3}+8 \mathrm{E}-05 \times$ tempo $^{2}$ $+0,0266 \times$ tempo $+2,3083)$

O volume mostrou uma correlação positiva entre momentos pré-estabelecidos da criação do pneumoperitônio (gráfico 2) e a curva mostrou um ajuste forte, com coeficiente de explicação igual a 0,96 (volume $=0,0157 \times$ tempo $+0,1813$ )

A previsão de PI e TVI (Tabela 2) mostrou: PI (mmHg): $1 \mathrm{~min}=4,15 ; 2 \mathrm{~min}=6,27 ; 3 \mathrm{~min}=8,36 ; 4 \mathrm{~min}=10,10$ e TVI (L): $1 \mathrm{~min}=1,12 ; 2 \mathrm{~min}=2,07 ; 3 \mathrm{~min}=3,01 ; 4 \mathrm{~min}=3,95$ e as médias reais e respectivos desvio padrão para PI e TVI foram : PI ( $\mathrm{mmHg})$ : $1 \mathrm{~min}=4,34(1,48) ; 2 \mathrm{~min}=6,05(1,44) ; 3 \mathrm{~min}=8,44(1,47) ; 4 \mathrm{~min}$ $=10,43(1,48)$ e TVI $(\mathrm{L}): 1 \mathrm{~min}=1,11(0,14) ; 2 \mathrm{~min}=2,18(0,21)$; $3 \min =3,13(0,27) ; 4 \min =3,87(0,40)$ 


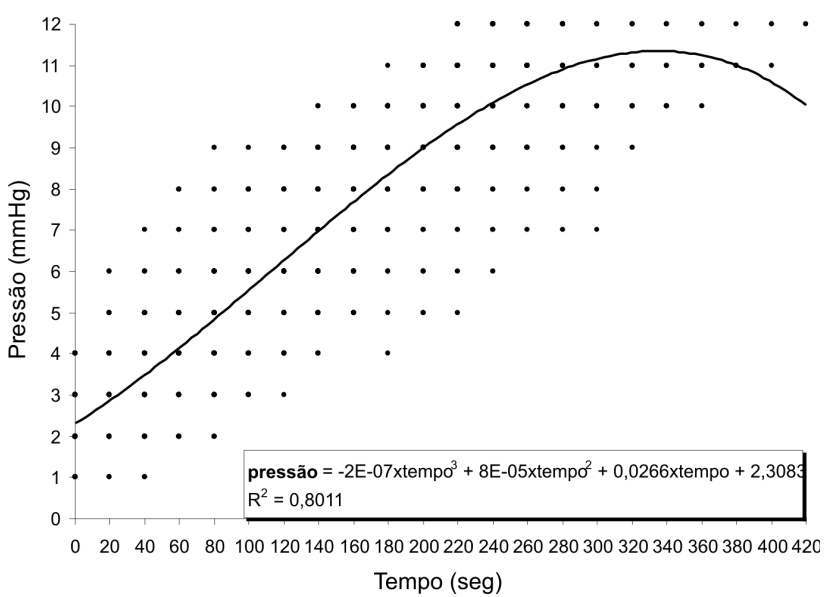

Gráfico 1 - Dispersão da pressão segundo o tempo de observação e respectiva curva de regressão. O modelo de regressão acima mostrou-se estatisticamente significante $(p<0,001)$ e com um coeficiente de explicação de $80,1 \%\left(R^{2}=0,8011\right)$, mostrando um bom ajuste.

\section{DISCUSSÃO}

A realização deste ensaio clínico prospectivo visa estabelecer parâmetros fidedignos para a orientação do cirurgião quanto ao real posicionamento da ponta da agulha de Veress após a punção, durante a criação do pneumoperitônio, provendo dados objetivos suficientes para avaliar o bom andamento (ou não) desse processo.

Para isto, momentos pré-determinados durante a criação do pneumoperitônio foram correlacionados à PI e ao VI, objetivando-se o estabelecimento de paradigmas quanto aos valores que devem ser esperados para aquelas variáveis dependentes (PI e VI) em função da variável independente (momento da insuflação considerado), expressando-se essa relação de forma matemática, mediante o estabelecimento de uma equação capaz de interligar as variáveis.

O coeficiente de correlação é um número puro, utilizado para classificar a correlaçao em perfeita $(=1)$, forte $(>0,75$ $\mathrm{e}<1)$, média $(>0,5 \mathrm{e}<0,75)$, fraca $(<0,5)$ e inexistente $(=0)^{12}$. Nesta pesquisa, tanto a PI quanto o VI apresentaram correlação com ajuste forte.

O insuflador para videocirurgia é o equipamento responsável pelo aporte de gás para a transformação das cavidades e espaços corpóreos virtuais em reais, com o propósito de propiciar a realização de procedimentos diagnósticos ou terapêuticos, mediante a criação de câmera de gás capaz de

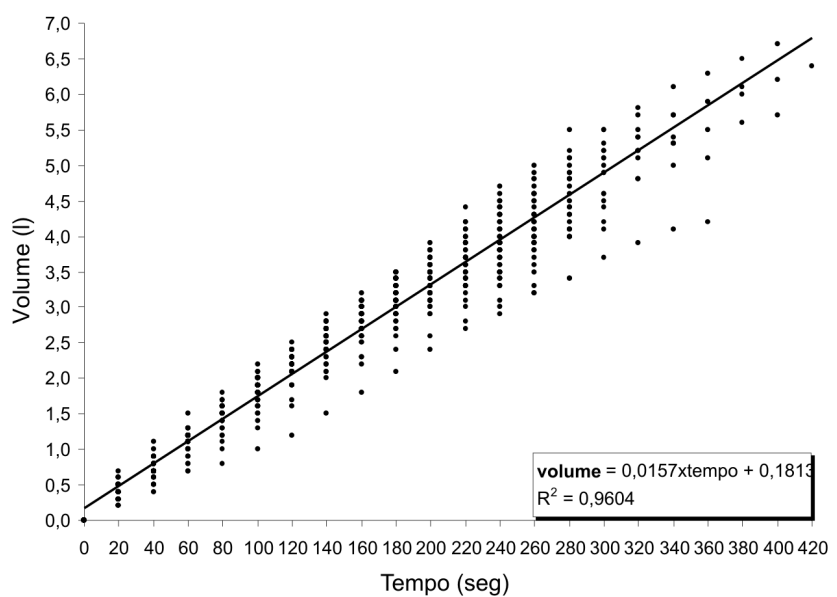

Gráfico 2 -Dispersão do volume segundo o tempo de observação e respectiva reta de regressão. $O$ modelo de regressão acima mostrou-se estatisticamente significante $(p<0,001)$ e com um coeficiente de explicação de $96,04 \%\left(R^{2}=0,9604\right)$, mostrando um ótimo ajuste.

prover clara visibilidade das estruturas anatômicas e adequada manipulação dos instrumentos videocirúrgicos. O equipamento possui programação que o torna simples e completamente automático. Toda vez que é ligado realiza um auto-teste, monitorando diversos pontos básicos do circuito eletrônico. Em caso de falha, o insuflador emite alarmes visual e sonoro, bloqueando o fornecimento do gás.

O valor da pressão estática é ajustável de 0 a $30 \mathrm{mmHg}$. $\mathrm{O}$ ajuste de pré-seleção é feito de modo fácil e rápido, através de comando nos botões do painel frontal. O circuito de pressão permite a medição de valores de pressão negativos, permitindo ao usuário, a realização de testes antes de iniciar a insuflação. O valor de fluxo é ajustável de 0 a $40 \mathrm{~L} / \mathrm{min}$. O valor do fluxo não assume imediatamente o valor pré-selecionado, mas aumenta gradativamente. O fluxo é pulsante e controlado eletrônicamente. Incorporado ao equipamento, há um totalizador de volume, indicando a quantidade de gás foi insuflada no paciente em determinado momento. Circuitos de segurança, mecânicos e eletrônicos, fornecem alto grau de proteção para o paciente. Os insufladores objetivam atingir a pressão intraperitoneal pré-estabelecida. Define-se também um limite superior para o fluxo. No início da insuflação, o equipamento mede a pressão tecidual transmitida pelo orifício da agulha e, caso essa seja menor que o limite máximo pressórico pré-fixado, começa a infundir gás. Nesta pesquisa, o valor do fluxo $(1,2 \mathrm{~L} / \mathrm{min})$ foi o sabidamente adequado à criação gradativa

Tabela 2 - Previsão dos valores de pressão e volume nos principais momentos de insulflação utilizando modelos estimados (pressão $=2,3083+0,0266 \times$ tempo $+8,3 \times 10^{-5} \times$ tempo $^{2}-2,44 \times 10^{-7} \times$ tempo $^{3} ;$ volume $=0,813+0,0157 \times$ tempo). Médias da pressão real, médias da pressão estimada e respectivos desvio padrão.

\begin{tabular}{lcccc}
\hline $\begin{array}{l}\text { Tempo de observação } \\
\text { em segundos }\end{array}$ & $\begin{array}{c}\text { Média real Pressão } \\
\text { (mmHg) e desvio padrão }\end{array}$ & $\begin{array}{c}\text { Média estimada } \\
\text { Pressão (mmHg) }\end{array}$ & $\begin{array}{c}\text { Média real Volume (L) } \\
\text { e desvio padrão }\end{array}$ & $\begin{array}{c}\text { Média estimada } \\
\text { Volume (L) }\end{array}$ \\
\hline 1 minuto & $4,34(1,48)$ & 4,15 & $1,11(0,14)$ & 1,12 \\
2 minutos & $6,05(1,44)$ & 6,27 & $2,18(0,21)$ & 2,07 \\
3 minutos & $8,44(1,47)$ & 8,36 & $3,13(0,27)$ & 3,01 \\
4 minutos & $10,43(1,48)$ & 10,10 & $3,87(0,40)$ & 3,95 \\
\hline
\end{tabular}


do pneumoperitônio, destarte minimizando nociva hiperreflexia do sistema nervoso parassimpático nocivos e dando valores exatos para volume esperado em litros por segundo ( $0,2 \mathrm{~L} / \mathrm{s})$. Quando a pressão medida começa a se aproximar da ajustada na máquina o fluxo pulsante começa a diminuir a fim de que a pressão desejada não seja ultrapassada.

Não há descrições na literatura sobre a evolução pressórica ou volumétrica do $\mathrm{CO} 2$ injetado durante a instalação do pneumoperitônio.

Nesta pesquisa a pressão e o volume permitiram ao pesquisador prever com segurança os valores em momentos chaves da insulflação (Tabela 2). Arbitrando-se momentos em 1, 2, 3 e 4 minutos, utilizando-se as equações de previsão da pressão e do volume deduzidas nas curvas de regressão (Gráficos 1 e 2), a pressão e o volume mostraram um ajuste forte.

Dessa forma, a observação das pressões intraperitoneais e dos volumes injetados em determinados momentos ao longo da insuflação com agulha de Veress na criação do pneumoperitônio artificial são parâmetros eficientes para a certificação da adequada localização intraperitoneal da ponta do instrumento.

\begin{abstract}
Background: To evaluate the possibility of establishing reliable parameters for the appropriate positioning of Veress needle tip in the peritoneal cavity during pneumoperitoneum creation. Method: In 100 selected patients Veress needle tip were introduced in the peritoneal cavity and the insufflators were programmed for a flow of 1,2L/min and with final maximum pressure of $12 \mathrm{mmHg}$. At the beginning of the insufflation and at every 20 seconds the intraperitoneal pressure (IP) and the total volume injected (TVI) were recorded. Data was treated by statistical correlation between moments and IP, and moments and TVI. The forecast values of IP and TVI at the end of each one of the four first insufflation minutes were also established, using the

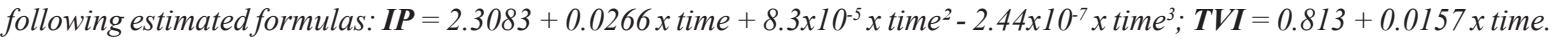
Results: IP and TVI showed a correlation between pre-established moments of pneumoperitoneum creation when a strong adjustment became apparent: IP $=-2 E-07 x$ time $^{3}+8 E-05 x$ time $^{2}+0.0266 x$ time +2.3083 , with a coefficient of explanation: $(R 2)=0.8011 ; \boldsymbol{T V I}=0.0157 x$ time +0.1813 , with $R 2=0.9604$. The forecast of $\boldsymbol{I P}$ and $\boldsymbol{T V I}$ showed: $\boldsymbol{I P}(\mathrm{mmHg})$ : $1 \min =4.15 ; 2 \min =6.27 ; 3 \min =8.36 ; 4 \min =10.10$ and $\boldsymbol{T V I}(L): 1 \min =1.12 ; 2 \min =2.07 ; 3 \min =3.01 ; 4 \mathrm{~min}=3.95$. Conclusion: Reliable parameters for IP and TVI can be established during pneumoperitoneum creation when the Veress needle tip is located in the peritoneal cavity in a given insufflation moment.
\end{abstract}

Key Words: Laparoscopy/adverse effects; Surgical procedures, operative; Pneumoperitoneum, artificial; Punctures/methods; Punctures/adverse effects.

\section{REFERÊNCIAS}

1. Bonjer HJ, Hazebroek EJ, Kazemier G, Giuffrida MC, Meijer WS, Lange JF. Open versus closed establishment of pneumoperitoneum in laparoscopic surgery. Br J Surg. 1997:84(4):599-602.

2. Baadsgaard SE, Bille S, Egeblad K. Major vascular injury during gynecologic laparoscopy. Report of a case and review of pubished cases. Acta Obstet Gynecol Scand. 1989;68(2):283-5.

3. Roviaro GC, Varoli F, Saguatti L, Vergani C, Maciocco M, Scarduelli A. Major vascular injuries in laparoscopic surgery. Surg Endosc. 2002;16(8):1192-6. Epub 2002 May 7.

4. Catarci M, Carlini M, Gentileschi P, Santoro E. Major and minor injuries during the creation of pneumoperitoneum. Amulticenter study on 12,919 cases. Surg Endosc. 2001;15(6):566-9. Epub 2001 Apr 3.

5. Schafer M, Lauper M, Krahenbuhl L. Trocar and Veress needle injuries during laparoscopy. Surg Endosc. 2001;15(3):275-80. Epub 2000 Dec 12.

6. Hanney RM, Alle KM, Cregan PC. Major vascular injury and laparoscopy. Aust N Z Surg. 1995;65(7):533-5.

7. Peterson HB, Greenspan JR, Ory HW. Death following puncture of the aorta during laparoscopic sterilization. Obstet Gynecol. 1982;59(1):133-4.

8. Chapron CM, Pierre F, Lacroix S, Querleu D, Lansac J, Dubuiosson JB. Major vascular injuries during gynecologic laparoscopy. J Am Coll Surg. 1997;185(5):461-5.

9. Fruhwirth J, Koch G, Mischinger HJ, Werkgartner G, Tesch NP. Vascular complications in minimally invasive surgery. Surg Laparosc Endosc. 1997;7(3):251-4.
10. Bergqvist D, Bergqvist A. Vascular injuries during gynecologic surgery. Acta Obstet Gynecol Scand. 1987;66(1):19-23.

11. Azevedo JLMC, Guindalini RSC, Azevedo OC, Paiva VC, Delorenzo A, Moreira MB. Avaliação do posicionamento da agulha de Veress durante o estabelecimento do pneumoperitônio pela técnica fechada, em porcos. Rev Col Bras Cir. 2004;31(5):318-23.

12. Guyatt G, Walter S, Shannon H, Cook D, Jaeschke R, Heddle N. Basic statistics for clinicians: 4. Correlation and regression. CMAJ. 1995;152(4):497-504.

Como citar este artigo:

Azevedo OC, Azevedo JLMC, Sorbello AA, Miguel GPS, Guindalini RSC, Godoy AC. Parâmetros eficientes do posicionamento adequado da ponta da agulha de Veress durante o estabelecimento do pneumoperitônio. Rev Col Bras Cir. [periódico na Internet]. 2006 Jul-Ago;33(4). Disponível em URL: www.scielo.br/rcbc

Endereço para correspondência:

Prof Dr João Luiz Moreira Coutinho de Azevedo

Universidade Federal de São Paulo - Setor de Videocirurgia

Prédio da Cirurgia Experimental

Rua Botucatu, 740 - Vila Clementino

04023-900 - São Paulo - SP

Telfax: (xx11) 5576-4272

E-mail: jozevedo.dcir@epm.br

Site: http//:www.cirurgiaonline.med.br 